\title{
Trayectorias globales: movilidad, procesos identitarios y nuevos aprendizajes en la capoeira Angola
}

\author{
Sergio González Varela \\ Doutor em Antropologia \\ Professor na Universidad Autónoma de San Luis Potosí, México \\ sergio.gonzalez@uaslp.mx
}

\begin{abstract}
Resumen: El presente artículo analiza el proceso de expansión global de la capoeira Angola enfocada principalmente en sus practicantes. El texto describe la movilidad de capoeiristas no brasileños y sus estrategias de aprendizaje, así como los itinerarios y trayectorias de los mestres de capoeira y otros miembros de las jerarquías de poder de los grupos alrededor del mundo. El artículo caracteriza a los practicantes internacionales como peregrinos de aprendizaje y a los mestres como misioneros culturales o de la tradición. Se argumenta que es en el encuentro entre ambos tipos de actores que el estilo Angola experimenta un proceso de resignificación como práctica afrobrasileña. A su vez, se hace hincapié en que la movilidad global de la capoeira implica un desarrollo de nuevas formas de aprendizaje que involucran una reflexión dialógica entre los conceptos de tradición e innovación. Finalmente, el texto concluye que la identificación de los dos tipos de actores descritos proporciona elementos importantes para el estudio antropológico de la movilidad y de la expansión global de la capoeira.
\end{abstract}

Palabras clave: Capoeira Angola; Movilidad; Globalización; Aprendizaje.

Abstract: This article analyzes the process of the global expansion of capoeira Angola, focusing on its practitioners. The text describes the mobility of non-Brazilian capoeira practitioners and their apprenticeship strategies, as well as the itineraries and trajectories of capoeira leaders (mestres) and other members of the groups' hierarchies of power around the world. The article characterizes international practitioners as apprenticeship pilgrims and the mestres as cultural or traditional missionaries. The argument states that it is in the encounter between these two actors that the Angola style is experiencing a resignification process as an Afro-Brazilian practice. At the same time, the text stresses that the global mobility of capoeira implies the development of new forms of learning that include a dialogue between the concepts of tradition and innovation. Finally, the article concludes that the identification of the two types of actors described provides essential elements to the anthropological study of mobility and the global expansion of capoeira

Key Words: Capoeira Angola; Mobility; Globalization; Apprenticeship. 


\section{Introducción}

El objetivo de este artículo es describir los diferentes tipos de movilidad que existen dentro del arte ritual de la capoeira Angola y analizar el impacto que dicha movilidad tiene en la construcción de nuevas identidades y en la resignificación de los conceptos de tradición e innovación del conocimiento. La estrategia es mostrar cómo la movilidad global de la capoeira depende de la distinción entre expertos brasileños (mestres, contramestres, profesores) y practicantes internacionales que buscan acumular más experiencia y conocimiento sobre la capoeira. A los expertos se les llamará misioneros culturales, esto debido al interés que los mestres y contramestres tienen de llevar el conocimiento de su tradición a los practicantes de capoeira alrededor del mundo, con el objetivo de difundir lo que ellos consideran como el verdadero sentido de este arte. A los practicantes globales que aspiran a acumular más conocimiento se les nombrará peregrinos de aprendizaje (apprenticeship pilgrims), un concepto que retomo de Lauren Griffith (2016) y su estudio sobre capoeiristas globales y sus experiencias de aprendizaje fuera y dentro de Brasil y de su trabajo en colaboración con Jonathan Marion (2018) donde ambos exploran de forma más detallada el concepto de peregrinaje de aprendizaje (apprenticeship pilgrimage) para otras artes corporales.

En este artículo, peregrinos de aprendizaje y misioneros culturales interactúan para dar cuenta de la movilidad global de la capoeira. En este encuentro el sentido de la capoeira Angola se transforma entrando en tensión elementos fundamentales de la definición identitaria de esta práctica, tales como los conceptos de tradición, innovación y ancestralidad. Los cambios de la capoeira, por lo tanto, ocurren tanto en los practicantes globales que aspiran a obtener un mayor conocimiento de este arte, como en los mestres quienes a raíz de sus experiencias fuera de Brasil han ido modificando sus discursos sobre la capoeira en estos últimos veinte años. Es en este proceso dialógico entre peregrinos y misioneros, que la capoeira Angola se muestra como un arte dinámica y en movimiento constante.

\section{El sentido global de la capoeira Angola}

La capoeira Angola ha experimentado una expansión global sin precedentes en los últimos veinte años. Este proceso no fue algo casual, es parte de una historia que comienza desde que Vicente Ferreira Pastinha, uno de los líderes más representativos de este estilo de capoeira, tuvo la oportunidad de viajar con algunos de sus alumnos y otras personalidades de la cultura afrobrasileña a la ciudad de Dakar en Senegal al Primer Festival de Artes Negras en 1966 (ASSUNÇÃO, 2005: 166). Este acontecimiento, aunado a la salida de capoeiristas de otros estilos desde la década de 1970 fuera de Brasil como miembros de compañías de danza contemporánea y la revitalización de la capoeira Angola en la década de 1980 y 1990, sentaron las bases de la movilidad mundial de practicantes de capoeira que vemos hoy en día.

En este proceso, es importante matizar que los tres estilos representativos de capoeira, a saber: Angola, Regional y Contemporánea ${ }^{1}$, marcan diferentes pautas y trayectorias que es preciso tomar en cuenta.

\footnotetext{
1 La diferencia entre estilos es parte de un proceso histórico producto de la formalización de la capoeira en academias. De esta manera, la capoeira Regional (llamada Luta Regional Baiana inicialmente), creada por Manoel Dos Reis Machado, Mestre Bimba en la década de 1930, se enfocó en su aspecto técnico, marcial y de competencia deportiva, arropada bajo una forma de identidad de carácter nacional. La capoeira Angola, surge en la década de 1940 en parte como un rechazo a la capoeira de Bimba y se centra en el rescate de las tradiciones afrobrasileñas y la herencia proveniente del pasado esclavista de Brasil. La capoeira contemporánea, surge en la década de 1970 como un intento de conciliación entre elementos deportivos, artísticos y marciales propios de la capoeira Regional y los elementos tradicionales provenientes de la capoeira Angola. Las tres capoeiras coexisten y engloban a la totalidad de los practicantes de capoeira a nivel mundial.
} 
No todos los estilos son iguales ni obedecen a objetivos y visiones comunes sobre el significado de la capoeira. Como lo ha mencionado Menara Guizardi en su trabajo sobre capoeiristas en Madrid, " dentro de cada uno de esos estilos de juego de la capoeira se construyen identidades relativas a la pertenencia a las diferentes agrupaciones" (GUIZARDI, 2017: 87). Es por esta razón, que en este artículo me concentro exclusivamente en la capoeira Angola como objeto de reflexión, ya que ha sido con miembros de este estilo con quien más he trabajado en mis investigaciones y porque al interior de los grupos, como lo menciona Guizardi, se forman identidades diferenciadas entre las tres modalidades de capoeira.

Algo distintivo de la expansión global dentro del núcleo de practicantes de la capoeira Angola es que no ha sido tan acelerado como en los otros dos estilos, sino que ha ocurrido de forma paulatina, esto debido a las propias características y exigencias de esta práctica, donde se privilegia la relación mestre-alumno como pilar fundamental del proceso de conocimiento y donde los líderes se asumen como los guardianes de la tradición. El concepto de tradición, enigmático y sin una definición clara y concisa dentro de los grupos de capoeira Angola, se posiciona como un elemento fundamental para entender los aspectos rituales y culturales de esta práctica. El antropólogo Celso de Brito, en un libro reciente sobre la globalización de la capoeira, menciona que la idea de tradición obedece a un proceso de construcción "validado a partir de disputas simbólicas entre varios agentes, incluyendo al Estado brasileño, capoeiras e intelectuales en dos contextos históricos específicos: 1930-40, y 1970-80" (BRITO, 2017: 41). En este proceso, otros elementos se vuelven parte importante en el desarrollo de la práctica de la capoeira como son la pertenencia a grupos legitimados dentro de una jerarquía de linajes bien identificada, la forma de ejecutar correctamente los movimientos corporales, la música y los gestos de acuerdo a las enseñanzas de un mestre (lo que los líderes llaman como Fundamentos) y elementos de carácter espiritual y religioso que son inherentes al estilo Angola.

Los elementos arriba mencionados: tradición, fundamentos, linajes, legitimidad, adherencia a seguir las enseñanzas de un mestre y un substrato espiritual, hacen que el desarrollo global de la capoeira tenga que pasar por estos filtros de calidad y control sobre la manera correcta de transmitir y enseñar la capoeira fuera de Brasil. La relación mestre-discípulo condiciona la forma en que la innovación ritual, corporal y musical ocurre en la práctica. La expansión global del estilo Angola, por lo tanto, se convierte en un proceso de negociación e inclusive de tensión entre practicantes internacionales y maestros en constante demanda moviéndose alrededor del mundo.

Dado que los mestres pueden ser considerados como ciudadanos globales, su rol como misioneros culturales los ha llevado en varios casos a migrar a otros países como han sido los casos de Mestre João Grande quien reside desde la década de 1990 en Nueva York, de Cobra Mansa quien ha residido en Washington D. C. y quien en años recientes ha regresado a vivir en Brasil y de otros mestres que viven en Europa como Mestre Laercio, Mestre Braga, Mestre Rosalvo y Mestre Marcelo Angola sólo por mencionar algunos casos representativos, la lista es bastante larga.

El interés por la movilidad de los expertos es correlativo al interés de practicantes no brasileños por aprender y saber más sobre la capoeira Angola. A este respecto, cabe decir que muchos grupos internacionales tienen una historia propia incluso de décadas con interacciones regulares con mestres brasileños y sus academias. Aunque este esquema que estoy describiendo es bastante general y simplificado, hay que tomar en consideración que la movilidad global dentro de la capoeira Angola tiene matices de todo tipo y escalas. Es decir, muchos practicantes internacionales tienen grados y lugares preponderantes en las jerarquías de poder de los grupos y linajes brasileños; algunos de ellos son profesores, contramestres o mestres e incluso han migrado temporalmente o permanentemente a Brasil. 
Por otro lado, los mestres muchas veces se desplazan a través de patrones de movilidad que han sido establecidos desde años atrás, con grupos filiales en diferentes ciudades del mundo y con relaciones sociales que se han expandido en los últimos años como consecuencia del internet, en lo que Menara Guizardi ha definido como la creación de redes sociales transnacionales (GUIZARDI, 2017: 155). En este escenario complejo de la movilidad también existen mestres que no tienen grupos, que incluso han rechazado viajar constantemente, o como el caso de Cobra Mansa o Boca do Rio, han regresado a vivir a Brasil después de largos periodos viviendo en el extranjero. En muchos sentidos, la movilidad de los mestres de capoeira Angola es de tipo diaspórico, como lo ha caracterizados Laurence Robitaille (2013: 144), donde los expertos salen de Brasil para viajar periódicamente a otros países o para migrar de forma permanente, pero siempre en contacto o hacia destinos que cuentan con comunidades afines a ellos, en este sentido forman parte de la diáspora afro-atlántica.

Muchos de los patrones de intersección de movilidad que se explicarán más adelante, también se replican en la relación mestre-discípulo dentro de Brasil, aunque con elementos diferenciales. Por lo tanto, el esquema simplificado de peregrinos de aprendizaje y mestres como misioneros culturales debe de ser entendido bajo esta óptica de complejidad donde existen un sinnúmero de variables entre ambos tipos agentes; este esquema es más una guía que una estructura rígida y que se posiciona con fines analíticos principalmente.

\section{La movilidad como eje argumentativo}

La antropología de la movilidad, lo que algunos han llamado el nuevo paradigma de la movilidad (SHELLER y URRY, 2006 \& URRY, 2007) obedece sobretodo a una perspectiva donde el movimiento y las dinámicas transformacionales definen el propio concepto de cultura. En este sentido, la movilidad no representa un giro radical de análisis, sino una atención a fenómenos de interconexión entre actores, lugares, flujos, dinámicas, elementos que siempre han estado en el análisis antropológico, pero que sólo recientemente han ido tomando un papel central en las discusiones de carácter teórico. Como lo señala el antropólogo Noel Salazar "los estudios sobre la movilidad llaman la atención a la infinidad de formas en las que la gente se vuelven parte, de forma desigual, de muchas redes y vínculos translocales" (SALAZAR, 2016: 2). Por lo tanto, el nuevo paradigma de la movilidad intenta ofrecer una mirada novedosa a un tema que siempre ha sido inherente a los grupos humanos: el movimiento constante de individuos y grupos fuera de los confines de sus entornos locales.

En su sentido filosófico, la movilidad puede entenderse desde el principio de multiplicidad establecido por Giles Deleuze y Felix Guattari (2002) quienes argumentan a favor de un plano de extensión inmanente donde las redes de conexión, las líneas de trayectoria son en sí mismas reveladoras, es decir, el plano de extensión de forma rizomática se vuelve la condición ontológica para dar cuenta de los procesos de multiplicación. Las características que definen al rizoma, tales como la heterogeneidad, las conexiones, la propia multiplicidad, las rupturas asignificantes, su modelamiento anti-estructural y de réplica y calco, dan la pauta para pensar una dinámica sin dirección univoca, sin principio y fin delimitado (DELEUZE y GUATTARI, 2002: 13-17).

Del mismo modo y aunque no directamente asociado con el nuevo paradigma de la movilidad, la obra de Bruno Latour (2005) sobre la teoría del actor-red resulta sugerente para entender las conexiones y redes que se generan a partir del movimiento de actores, ideas y objetos materiales a nivel global. Para Latour, la teoría del actor-red pasa por una redefinición de lo social, la cual "tiene que ser mucho más amplia que aquello a lo que generalmente se llama por ese nombre, pero estrictamente limitada al rastreo de nuevas asociaciones y al diseño de sus ensamblados" (LATOUR, 2005: 21). 
Dentro de su perspectiva, Latour ofrece una visión similar a la de Deleuze y Guattari sobre el plano de inmanencia y extensión que se contrapone al polo de trascendencia y abstracción del pensamiento que él identifica como piedra angular de la sociología clásica. Es decir, en ambas posturas, la de Latour y la de Deleuze y Guattari, se privilegia el sentido territorial, de dislocación en el cual operan los flujos y la movilidad, sin necesariamente tener que remitirse a un plano abstracto, vertical, trascendente y preexistente.

Lo que me interesa de las posturas del nuevo paradigma de la movilidad, la idea de multiplicidad y rizoma de Deleuze y Gauttari, y la teoría del actor-red de Latour es el esfuerzo por entender el movimiento de personas, objetos e ideas de manera extensiva, territorial, desarrollando una constante creación de relaciones. Los alcances de este tipo de visión, creo yo, son de utilidad para entender los procesos de expansión global de la capoeira Angola, los cuales ocurren en un plano de extensión que no obedecen a un solo patrón de movimiento, a una causa específica o a una motivación meramente individual. Las ideas de relación, multiplicidad y variabilidad sin una dirección específica, son índices de la manera en que el mundo actual se mueve, ya sea en los desplazamientos territoriales de las personas o en los imaginarios y dislocaciones producto del Internet y las redes sociales. En el caso de la movilidad de los practicantes de capoeira Angola, el rastreo de asociaciones en un plano extensivo resulta sugerente para dar cuenta de las trayectorias de expertos y estudiantes de manera que su explicación no se reduzca a una mera referencia anecdótica, sino que arrojen una manera diferente de entender los procesos dinámicos de expresión de una práctica ritual y de transformación de las identidades. Es a estos aspectos a los cuales se les dará atención a continuación.

\section{Peregrinos de aprendizaje}

Uno de los trabajos más interesantes, aunque no el único, sobre practicantes internacionales de capoeira es el de Lauren Griffith (2016). Si bien existen otros trabajos que se enfocan al estudio de la capoeira en su sentido global (ver BRITO, 2017, GUIZARDI, 2017; DELAMONT, STEPHENS y CAMPOS, 2017; HEDEGARD, 2012; ROSA, 2015 y WESOLOWSKI, 2012), el trabajo de Griffith resalta por su propuesta conceptual de caracterizar a los practicantes internacionales de este arte brasileño como peregrinos de aprendizaje. Este concepto, expandido posteriormente en su trabajo colaborativo con Jonathan Marion (2018), es de utilidad para comprender cómo se constituye la comunidad de practicantes de capoeira a nivel global. Una de las ventajas del modelo de Griffith es que se enfoca principalmente en practicantes del estilo Angola, lo que acota el contexto de análisis. Me parece que esta decisión es pertinente debido a lo que he señalado al principio del artículo sobre las complejidades de los otros dos estilos de capoeira para poder hablar de este arte ritual en un sentido general.

Lauren Griffith define al peregrinaje de aprendizaje como "el viaje al lugar de origen u otro lugar comúnmente ratificado como matriz de la práctica con la meta explícita de estudiar este arte o deporte bajo la tutela de un maestro local" (GRIFFITH, 2016: 16). Un peregrino de la capoeira, por lo tanto, es alguien que toma en serio esta práctica con el objetivo de aprender y ganar más conocimiento y legitimidad sobre ella. No es aquella persona que se acerca a tomar una clase de capoeira en Brasil o en otro país de forma casual. El peregrino se define como alguien comprometido por cierto tiempo al estudio y práctica de la capoeira y que viaja local, regional, nacional e internacionalmente por causa de este arte. Contrastando y ampliando el sentido de la peregrinación dado por Victor Turner (2009), Lauren Griffith argumenta que peregrinar no necesariamente tiene que estar restringido a prácticas religiosas. Un peregrino de la capoeira, como ella lo define, tiene el interés de aprender por cuestiones de habilidad, versatilidad y destreza, su motivación es secular (GRIFFITH, 2016: 60). 
Varios de los puntos que desarrolla Griffith sobre los practicantes internacionales de capoeira se refiere a gente que viven en los Estados Unidos, pero bien pudiera generalizarse a practicantes de otros países. Este ímpetu generalizador queda de manifiesto en su libro con Jonathan Marion donde el concepto de peregrinación de aprendizaje se extiende para entender otras prácticas deportivas y dancísticas, incluso otro tipo de comunidades que poco tienen que ver con prácticas corporales como coleccionistas, expos sobre comics, entre otras. En esta nueva colaboración, Griffith y Marion definen al peregrinaje de aprendizaje como una alternativa a la caracterización del término "turismo" que trastoca y subvierte las definiciones clásicas de lo que se entiende por este término (GRIFFITH Y MARION, 2018: xviii).

Un peregrino en este sentido, invierte tiempo, dinero y esfuerzo por causa de la capoeira y su interés es conocer más sobre este arte. Esto obliga al practicante a aprender el idioma portugués en la medida de lo posible, adentrarse en el reportorio musical de la capoeira, dominar los instrumentos de la orquesta musical, en especial el berimbau y desarrollarse más fluidamente en la práctica corporal; también se interesa en los aspectos históricos, religiosos y filosóficos de este arte. El peregrino adquiere parte de estos conocimientos con su profesor local, pero sobretodo en viajes regionales, nacionales y ultimadamente en Brasil. El peregrino de aprendizaje viaja para saber más de un arte, idealmente hacia su lugar de origen (GRIFFITH y MARION, 2018: xix).

En este viaje, el peregrino aspira a formar parte de una comunidad de practicantes y quiere ganarse el respeto y legitimidad en Brasil. La capoeira Angola proporciona al practicante un nuevo sentido de su identidad y de relacionarse con un nuevo entorno. Griffith y Marion describen con detalle las diferentes escalas y momentos del peregrinaje y comparan diferentes prácticas, incluso hablan de peregrinajes virtuales (GRIFFITH y MARION, 2018: 127:142).

Para los peregrinos de aprendizaje el objetivo ideal es viajar, aunque sea una sola vez, a Brasil. Brasil, en este sentido, se convierte en el destino último para el practicante internacional de capoeira Angola; ir a este país significa visitar la fuente de conocimiento y sabiduría de la capoeira. Tradicionalmente, este origen es en la ciudad de Salvador, estado de Bahía, donde presumiblemente la capoeira tiene sus fundamentos históricos. No obstante, actualmente el peregrinaje puede ser hacia el grupo matriz en otras ciudades como São Paulo, Brasilia, Rio de Janeiro entre otras, esto aplica sobretodo para practicantes internacionales que son miembros de grupos grandes filiales como lo son la Fundação Internacional de Capoeira Angola (FICA) o Grupo Nzinga que tienen academias fuertes no sólo en Salvador sino en otras ciudades.

El peregrinaje de estudiantes internacionales, como bien lo señala Lauren Griffith se mueve por diferentes escalas geográficas con la motivación ideal de viajar a Brasil. Este viaje puede que ocurra o que no ocurra. No todos los practicantes internacionales cuentan con los recursos financieros para hacerlo y para muchos implica un sacrificio. Los estudiantes con los que trabaja Griffith son estadounidenses y los peregrinos que ella encuentra en Salvador son o de este país o europeos. Habría que ver qué matices toma el concepto de peregrinaje de aprendizaje con practicantes de capoeira de otras latitudes que no cuentan con la infraestructura financiera para poder viajar a Brasil o para poder invitar regularmente expertos a sus países.

Aunque existen varios matices que complejizan en la práctica el concepto de peregrino de aprendizaje, su valor radica en caracterizar las dinámicas de movilidad de practicantes internacionales por motivos de la capoeira. En este sentido, se convierte en un concepto de utilidad para entender este tipo de movilidad dentro de los practicantes de un arte ritual de forma desterritorializada. Aunque el espíritu de este concepto deriva del peregrinaje como un proceso de aprendizaje de habilidades de tipo secular, es posible que conforme el peregrino pasa más tiempo involucrado en la capoeira se comience a tener otro sentido sobre el significado de la capoeira que, como lo veremos más adelante, puede derivar en un proceso educativo de tipo espiritual y religioso. Griffith y Marion apuntan hacia este tipo de peregrinaje más profundo, pero es algo que no desarrollan de manera detallada. 
Como veremos en la siguiente sección, este otro sentir del peregrinaje depende del otro lado de la moneda, es decir, el polo de los líderes y sus dinámicas de movilidad, que, de manera simultanea, rigen el destino de la expansión global de la capoeira. Es a estos actores a los que habrá que enfocarse a continuación.

\section{Los mestres como misioneros culturales}

Si por un lado tenemos a los practicantes de capoeira internacionales como peregrinos de aprendizaje, por el otro tenemos a los expertos como misioneros culturales. El concepto de peregrino de aprendizaje, como hemos señalado, es un concepto que emana de las dinámicas de movilidad de los practicantes no brasileños y que describe de manera eficaz el movimiento de personas interesadas por este arte. Pese su valor analítico, su definición ocurre de manera externa a la práctica de la capoeira, es decir, es una construcción conceptual que no surge de los propios practicantes, sino que es el resultado creativo e inventivo de Lauren Griffith. Esto no demerita en absoluto la validez de este concepto, pero etnográficamente no proviene de lo que algunos antropólogos denominarían como "el punto de vista del nativo". Este punto de vista local, lo encontraremos en la descripción de las dinámicas de movilidad de los expertos, de los líderes de la capoeira quienes se asumen como misioneros culturales, guardianes de la tradición y quienes tienen por objetivo llevar el “verdadero" mensaje sobre los fundamentos de la capoeira Angola.

A diferencia de los practicantes internacionales, los expertos son individuos, en su mayoría hombres, que tienen como misión mostrar al mundo la forma correcta de practicar la capoeira y de preservar esta tradición afrobrasileña. Como lo menciona Ilnete Porpino de Paiva, para ser considerado un mestre de capoeira "es necesario transmitir conocimiento" (PAIVA, 2007: 123). El interés de los mestres, contramestres y miembros de las jerarquías de poder en la capoeira tiene que ver con la difusión de un tipo de conocimiento que no puede ser transmitido exclusivamente de manera virtual por medio de Youtube y otras plataformas electrónicas. Aunque los líderes actualmente usan estos medios electrónicos para llegar a sus discípulos, la presencia física es vital para entender la transmisión del conocimiento de este arte.

Los mestres como misioneros culturales y guardianes de la tradición pueden ser considerados embajadores de la cultura afrobrasileña, aunque cabe decir que no todos los líderes de la capoeira son afrobrasileños. El tema racial y de política étnica no es para nada superfluo y se encuentra inserto dentro de las prácticas identitarias en Brasil y en otros contextos con poblaciones afro como Estados Unidos, Inglaterra y el Caribe, sólo por mencionar algunos lugares representativos. La capoeira Angola juega un papel importante en la construcción de una identidad diferenciada de tipo étnico-racial que se encuentra en los discursos de los líderes en Brasil y en sus viajes al exterior.

El contexto no es sencillo de entender y existen actualmente en Brasil debates y discusiones sobre quiénes pueden hablar o no en nombre de la capoeira, si el tema racial es esencial para definir su práctica, o si existen apropiaciones culturales de otros sectores sociales sobre su saber. Al ser la capoeira y el título de "Mestre de Capoeira" objeto de reconocimiento cultural patrimonial nacional por el Instituto do Patrimônio Histórico e Artístico Patrimonial (IPHAN) y de status global por la UNESCO (ver FONSECA y VIEIRA, 2014), los discurso sobre la autenticidad y la legitimación tradicional se han convertido en prácticas que orientan a la capoeira en Brasil cada vez más hacia un polo político e ideológico. Este debate étnico-político parece ser más fuerte dentro de los grupos de capoeira Angola que en otros estilos, aunque no es para nada nuevo. En el año 2011, la antropóloga Katya Wesolowski apuntaba ya a la existencia de discusiones sobre la autenticidad y legitimidad de la capoeira y sus estilos fuera de Brasil, donde por lo menos en Estados Unidos, se dirigían a temáticas de corte racial (WESOLOWSKI, 2011:88). 
Las discusiones raciales, sobre orígenes africanos y el esencialismo que muchos líderes y académicos profesan sobre la capoeira Angola son parte de su práctica a nivel mundial y no pueden dejar de pasar desapercibidas. Lo que me interesa en esta sección es mostrar cómo el ser misionero está cambiando la manera en que los mestres transmiten su conocimiento a las nuevas generaciones en otros países, su repercusión en sus grupos en Brasil y sus relaciones sociales que establecen entre ellos y con miembros no brasileños dentro de las jerarquías de la capoeira Angola.

En un sentido estrictamente corporal, la presencia física de un mestre, el que haya viajado de un lugar lejano o salido de gira para ofrecer sus conocimientos, simboliza no sólo su compromiso, sino también su poder, su maestría y dominio de este arte. Las enseñanzas pueden ser mínimas o demasiado simples, muchas veces el conocimiento de un líder pasa por su manera de tocar la música, de cantar y de moverse y no por presentar secuencias corporales complicadas. A este respecto, algo tan básico en la capoeira como el movimiento de la ginga se transforma en un proceso profundo de internalización de una tradición afrobrasileña. Como lo ha señalado Cristina Rosa, el aprendizaje de la ginga se convierte en una coreografía estética de procesos de identificación, con multiniveles de expresión y representación (ROSA, 2015: 5-8). Este aprendizaje forma parte de las enseñanzas que un líder muestra a sus discípulos fuera de Brasil y que el peregrino de aprendizaje difícilmente puede obtener con su profesor local (al menos que este sea un mestre brasileño, lo cual llega a pasar sólo en contadas ocasiones).

Los viajes internacionales de los mestres son de tipo rizomático, es decir, no obedecen a un único patrón de movilidad, se multiplican por el orbe y algunas veces duran desde pocos días hasta meses enteros. Muchos mestres salen al exterior en su función de misioneros y guardianes de la tradición y sus itinerarios con frecuencia cambian y nuevos destinos se añaden a los ya agendados de forma un tanto espontánea, esto sin mencionar que los líderes también poseen una agenda de viajes regulares dentro de Brasil. El tipo de movilidad que estos líderes ejecutan, si es posible hablar de una direccionalidad, es de Brasil hacia fuera, mientras que el de los peregrinos de aprendizaje tiene como objetivo en algún momento viajar a Brasil. Ambas formas de movilidad son opuestas, pero se intersectan, más adelante ahondaré en las diversas formas de encuentros entre mestres y practicantes internacionales, por el momento quisiera continuar describiendo los procesos de movilidad de los mestres de capoeira Angola.

Al ser ciudadanos globales, los expertos de la capoeira han creado mecanismos de diferenciación con respecto al resto de los practicantes de la capoeira Angola. Como ya lo he mencionado anteriormente, el que los líderes se consideren a sí mismos como los guardianes de la tradición y los misioneros de la cultura afrobrasileña define el tipo de relaciones y encuentros que tienen con los practicantes internacionales. Debido a que la destreza física y la práctica corporal pueden ser imitadas o desarrolladas a lo largo de los años de manera casi experta por parte de practicantes extranjeros y que su dominio musical puede ser casi perfecto, la singularidad de los mestres como figuras de poder y autoridad pasa por el tema de lo que ellos definen como los fundamentos de la tradición y como el conjunto de saberes que ellos detentan y que no sólo depende de la destreza física o la habilidad musical. Los expertos tienen poder, el cual ha sido aprendido y desarrollado con los años, tienen mandinga, incorporan la malandragem y la astucia de manera tal que sus formas de expresión son muy diferentes al del resto de los practicantes, es eso lo que Cristina Rosa ha descrito con detalle sobre la parte coreográfica de identificación y aprendizaje de la ginga y otros movimientos corporales dentro de una lógica de juego que parece no tener ninguna lógica, como lo menciona Floyd Merrel (2005). En sus viajes, los mestres de capoeira Angola intentan mostrar cómo podría ser una vida dedicada a la capoeira si los practicantes no brasileños tomaran los fundamentos de la tradición seriamente hasta sus últimas consecuencias. 
En este proceso global de transmisión de conocimiento, los mestres también necesitan protegerse, es decir, no pueden darlo todo a los practicantes con los que interactúan, sobre todo con gente de otros grupos y linajes. Entonces, por un lado, tenemos a expertos preocupados por difundir las enseñanzas de la capoeira Angola, pero, por otro lado, tenemos a individuos recelosos de revelar todos los secretos y tradiciones de este arte. Esta forma de autopreservación, de mecanismo de poder para dar conocimiento a otros de forma dosificada sigue precisamente los dictados cosmológicos de otras prácticas afrobrasileñas como es el caso del Candomblé, donde el conocimiento de esta religión es paulatino, lento y como dice el antropólogo Márcio Goldman (2008), tiene que ver con recoger los trozos de conocimiento que indirectamente los líderes espirituales dejan a sus discípulos y donde las preguntas directas son consideradas de mala educación. Esta forma de relacionarse entre expertos y discípulos es característica de otras prácticas culturales como la hechicería en donde muchas veces el aprendiz no recibe instrucciones verbales claras y directas sobre dichas prácticas de parte de los hechiceros, como lo fue en el caso de Paul Stoller (STOLLER y OLKES, 1987) y su vivencia de aprendiz de hechicero con Adamu Jenitongo en Niger.

De lo anterior se deduce que los mestres de capoeira viajan para difundir su conocimiento, para tener fuentes de ingresos para sus familias, por curiosidad, por compromiso y también por diversión. No obstante, sus relaciones con practicantes fuera de Brasil y con miembros de otros linajes son cautelosas y con cierta reserva sobre el grado de revelación de conocimiento sobre su tradición y el vínculo que tengan con otros practicantes. Algunas explicaciones que los mestres han dado al respecto tienen que ver con el tipo de conocimiento a largo plazo que la capoeira implica. Para los mestres, es importante hacerles ver a los peregrinos de aprendizaje que la capoeira es una forma de vida que se extiende por varios periodos de sus vidas y que no se puede dominar en un corto plazo o restringirse al aprendizaje de habilidades meramente físicas; es un camino arduo y difícil. El mismo Mestre Pastinha afirmaba que la capoeira era un proceso arduo, difícil, lleno de traiciones y desafíos y que al final uno se daba cuenta que una vida no era suficiente para comprenderla.

Los mestres tienen muy presente que el arte al cual dedican sus vidas es complejo y que, al ser una práctica holista, siempre rebasará sus expectativas y su dominio. Sus viajes, su forma de ser seminómada en la actualidad, ofrecen un tipo de movilidad extensivo, con líneas de fuga que trazan asociaciones y ensamblajes a nivel global y que no se pueden entender bajo un solo patrón. En la siguiente sección veremos cómo es que se lleva a cabo la interacción entre peregrinos de aprendizaje y los misioneros culturales en estas dimensiones de la movilidad actual.

\section{Encuentros transversales y nuevos aprendizajes}

Los peregrinos de aprendizaje y los misioneros culturales tienen diferentes expectativas y posiciones sobre lo que constituye la alteridad. Su encuentro es muy similar al que existe entre el antropólogo y el otro, que, como lo señala Roy Wagner, es crucial en la antropología, donde coexiste un proceso dialógico y recursivo entre dos formas de inventar otras realidades y de asimilarlas a las convenciones en las cuales viven (WAGNER, 1981). Es en el encuentro, en la relación que se establece entre el antropólogo con el otro, que el proceso creativo del trabajo de campo emerge y da pie a la descripción etnográfica. Eduardo Viveiros de Castro ha señalado que este encuentro suscita un horizonte comparativo de traducción, aunque advierte que el sentido de lo que se entiende por comparación debe de ser otro, menciona que: "la comparación de la cual hablo es una regla constitutiva de la disciplina. Atañe al proceso involucrado en la traducción de los conceptos prácticos y discursivos del nativo en los términos del aparato conceptual de la antropología" (VIVEIROS DE CASTRO, 2015: 57). 
Para este autor, traducir implica controlar las equivocaciones que se puedan tener entre la relación del antropólogo con el otro, ya que traducir siempre implica perder algo del sentido original de lo que se traduce. Este encuentro tiene implicaciones no sólo epistemológicas sino ontológicas.

En el caso que nos compete, el peregrino de aprendizaje proviene de una cultura diferente a la de los misioneros o vive en un contexto cultural distinto al de ellos y los procesos de traducción son de carácter transversal. Los encuentros entre mestres y estudiantes no brasileños pueden generar confusiones y malentendidos. Es decir, los expertos pueden sentir que su mensaje no está siendo asimilado y entendido como ellos quisieran. A su vez, los practicantes no brasileños pueden asumir de forma equivocada el sentir y los discursos de un líder e inclusive estar en total desacuerdo con sus enseñanzas. La situación se complica, ya que, como parte del proceso global de expansión de la capoeira, varios mestres han incorporado nuevos elementos en sus enseñanzas sobre el significado del concepto de "tradición" y han llevado las discusiones etno-políticas sobre lo que es ser afrobrasileño a contextos fuera de Brasil. Otros han hecho énfasis en el aspecto espiritual y religioso de la capoeira Angola de forma más pronunciada.

La alta demanda de expertos a nivel mundial y su reconocimiento de prestigio como guardianes de la tradición también afecta la manera en que los peregrinos de aprendizaje perciben a dichos expertos. No es algo aislado el que miembros internacionales muestren más adherencia a los dictados de la tradición y de forma más comprometida que sus contrapartes en Brasil, como lo ha notado Celso de Brito en su descripción etnográfica con grupos franceses de capoeira en Lyon, donde los fundamentos sobre la tradición y religiosidad afrobrasileña son bastante pronunciados (BRITO, 2017). También puede llegar a suceder que estudiantes internacionales otorguen a la figura del mestre de capoeira un aura sagrada y de misticismo ligada a una veneración de tipo espiritual, que puede que esté ausente en Brasil. El experto, a su vez, puede realzar su papel como educador y misionero a tal grado que es posible que asuma ese rol místico y espiritual que se le adjudica en el extranjero.

Son muchas las situaciones que caracterizan la relación mestre-estudiante en un contexto de movilidad. Existen expectativas de todo tipo, fascinación y agradecimiento por las enseñanzas recibidas y la oportunidad por parte de los mestres de poder compartir su conocimiento, aunque también existen desilusiones, desencantamientos y desavenencias. El contexto en el que ocurren estos encuentros se da en los llamados talleres o workshops que grupos internacionales realizan con regularidad para invitar a sus mestres y expertos de otros linajes. En los talleres o encuentros internacionales, coinciden decenas de practicantes de varias regiones y países. Son momentos de peregrinaje donde capoeiristas definen y confirman sus identidades y donde enriquecen su saber sobre la capoeira. Para los mestres, los encuentros son momentos para transmitir sus conocimientos, sus versiones sobre los fundamentos del estilo Angola y su saber ancestral. Los workshops ofrecen un momento de interacción entre misioneros y peregrinos, donde viejos amigos se encuentran, donde nuevas relaciones de amistad emergen y donde, en ocasiones, viejas rivalidades se reavivan. Las reuniones en talleres intensivos fuera de Brasil muchas veces reúnen una cantidad significante de mestres de diversos linajes quienes quizá poco o nulo contacto tienen entre ellos en Brasil. En los grandes centros europeos de la capoeira Angola como Londres y Berlín, por ejemplo, los talleres son más recurrentes y el flujo de mestres visitantes es más continuo que en otras regiones. En otros países, sobretodo no pertenecientes al primer mundo, la oportunidad de interactuar con un mestre reconocido es menor, ya que traerlo implica un gasto bastante fuerte para los grupos locales.

En su sentido estrictamente antropológico, la interacción entre expertos y practicantes internacionales ofrece un encuentro entre perspectivas, entre culturas y procesos oblicuos y transversales, donde no sólo puede haber problemas de interpretación, sino también diferencias ontológicas y hasta cosmológicas. 
El sentido de la capoeira Angola para un mestre es completamente diferente al que va adquiriendo un estudiante internacional; los procesos de aprendizaje de ambos son de otra índole y el hecho de que dicho proceso no esté ocurriendo cotidianamente en Brasil y con cierta regularidad, hace que la transmisión del conocimiento pase por estas inconstancias temporales.

Los encuentros globales entre los expertos y los practicantes no brasileños también dependen de cierta comercialización de la capoeira donde el mestre es el artífice de su propia “empresa" para usar el término que Laurence Robitaille (2013: 166) utiliza para caracterizar las relaciones comerciales en la capoeira. En esta comercialización, el conocimiento que posee un mestre tiene un valor de mercancía y está inserto dentro de una política comercial capitalista. Esto no impide que los líderes puedan compaginar este interés económico con su papel de misioneros y guardianes de la tradición; ambas opciones no están peleadas. Actualmente, ningún mestre que yo conozca me ha compartido el deseo de terminar sus días como el mítico Mestre Pastinha, en pobreza extrema, ciego y en el olvido. Al contrario, los líderes quieren solventar sus dificultades económicas, desean incrementar sus ingresos y llevar su mensaje sobre los fundamentos de la capoeira a todo el mundo.

En el caso de los peregrinos de aprendizaje, hay que recalcar que no todos tienen el mismo compromiso y dedicación con la capoeira, esto varía dependiendo de sus motivaciones y el que hayan aceptado o no un cargo de responsabilidad dado por un mestre con el cual están asociado. Los practicantes que viven fuera de Brasil, al asumir el control de un grupo en representación de un mestre, están a su vez integrándose dentro de las jerarquías y los linajes brasileños a los que sus mestres pertenecen. Lo mismo aplica para practicantes en Brasil, donde el compromiso de los miembros de un grupo varía con el paso del tiempo, muchos desisten, otros rechazan cargos, una minoría asumen puestos en las jerarquías de poder y finalmente sólo unos pocos llegarán a convertirse en mestres.

Pese a todas las variaciones que se puedan encontrar en la relación que se establece entre un mestre y sus discípulos, lo que encontramos son formas de conexión que han ido cambiando la manera de entender el concepto de tradición y saber dentro del estilo Angola con influencias de todo tipo, como por ejemplo el uso de las técnicas de la capoeira para desarrollar una pedagogía libertaria (MATA, 2001), la existencia de grupos espirituales ligados al New Age y la mezcla de las tradiciones afrobrasileñas con las tradiciones locales de otros países. Todas estas y otras manifestaciones, son producto en parte de las dimensiones antropológicas de la movilidad y de la expansión global de la capoeira Angola.

\section{Conclusión}

Las trayectorias globales de la capoeira Angola pasan por la identificación de dos tipos de actores sociales: los peregrinos de aprendizaje y los misioneros culturales. Ambos actores definen los procesos de movilidad de este arte afrobrasileño y orientan la forma en que las relaciones sociales se establecen entre ellos. Este encuentro entre peregrinos (practicantes fuera de Brasil) y los misioneros (los expertos de la capoeira) es en muchos sentidos la coincidencia de perspectivas divergentes y de miradas transversales que no siguen los mismos objetivos. Para un mestre, la capoeira Angola es su vida, es su trabajo y su dedicación es exclusiva. Para un peregrino de aprendizaje, la capoeira es importante, pero no dedica su vida completamente a ella y raras veces se le paga para que se convierta en un misionero cultural, la contrario, él paga y viaja para aprender de un mestre.

La identificación de los dos tipos de actores en este artículo, caracterizan patrones de movilidad múltiples que no se dirigen hacia los mismos fines. Para un peregrino, el destino último es viajar a Brasil. Para un mestre su destino es hacia fuera de Brasil, su interés es llevar su mensaje de la tradición a todo el mundo. Las identidades de ambos actores se modifican a partir de su encuentro mutuo. 
Un mestre aprende de sus estudiantes extranjeros a la vez que estos últimos aprenden sobre los fundamentos de la capoeira Angola de sus líderes. En esta interacción, un proceso creativo y sutil toma forma, donde nuevos sentidos de la capoeira Angola se discuten y negocian. Como parte de este intercambio de perspectivas, novedosas maneras de interactuar en el mundo surgen y los horizontes de ambos actores se amplían y retroalimentan.

Dentro del marco de los estudios antropológicos, históricos y sociales de la capoeira en general, es importante notar que hay mucho trabajo por hacer en cuanto a temas de investigación. La expansión global de la capoeira, la emergencia de estudios sobre este tema y las investigaciones que se están llevando a cabo en Brasil, nos habla de un corpus de referencias en diferentes idiomas, cada vez más amplio y que sigue creciendo a nivel mundial en forma de artículos, libros y tesis de licenciatura, maestría y doctorado. Es, por tanto, imprescindible que los elementos señalados en este artículo como la movilidad, los actores descritos y sus trayectorias globales, sean reforzados o matizados a partir de nuevas investigaciones de campo en lugares donde todavía no se cuenta con investigaciones detalladas. Sólo así, es que los estudios académicos sobre la capoeira darán cuenta de su dimensión global y desterritorializada.

\section{Referencias bibliográficas}

ASSUNÇÃO, M. R. Capoeira: The History of an Afro-Brazilian Martial Art. London: Routledge, 2005.

BRITO, C. A Roda do Mundo: A Capoeira Angola em Tempos de Globalização. Curitiba: Appris Editora, 2017.

DELAMONT, S; STEPHENS N. y CAMPOS C. Embodying Brazil: An Ethnography of Diasporic Capoeira. London: Routledge, 2017.

DELEUZE, G. y GUATTARI, F. Mil mesetas: Capitalismo y esquizofrenia. Valencia: Pre-Textos, 2002.

FONSECA, V. L., y VIEIRA, L. R. “Capoeira-A Brazilian Immaterial Heritage: Safeguarding Plans and Their Effectiveness as Public Policies". International Journal of the History of Sports 31 (10), 2014: 1303-11.

GOLDMAN, M. "How to Learn in an Afro-Brazilian Spirit Possession Religion: Ontology and Multiplicity in Candomblé". In: Learning Religion: Anthropological Approaches, BERLINER, D y SARRÓ, R. New York: Berghahn Books, 103-19, 2008.

GRIFFITH, L. M. In Search of Legitimacy: How Outsiders Become Part of the Afro-Brazilian Capoeira Tradition. New York: Berghahn Books, 2016.

GRIFFITH, L. M. y MARION, J. Apprenticeship Pilgrimage: Developing Expertise through Travel and Training. LANHAM, H. W. Boulder, New York and London: Lexington Books, 2018.

GUIZARDI, M. Capoeira: Etnografía de una historia transnacional entre Brasil y Madrid. Santiago de Chile: Ediciones Universidad Alberto Hurtado, 2017. 
HEDEGARD, D. "Becoming a Capoeirista: A Situational Approach to Interpreting a Foreign Cultural Good". Sociological Inquiry 82 (4): 510-31, 2012.

LATOUR, B. Reensamblar lo social: Una introducción a la teoría del actor-red. Buenos Aires: Manantial, 2005.

MATA, J. A liberdade do Corpo. Soma, capoeira angola e anarquismo. São Paulo: Editora Imaginário.

MERRELL, F. Capoeira and Candomblé: Conformity and Resistance through Afro-Brazilian Experience. Princeton, NJ: Markus Wiener, 2005.

PAIVA, I. P. A capoeira e os mestres. Dissertação de doutorado: Universidade Federal do Rio Grande do Norte, 2007.

ROBITAILLE, L. Capoeira as a Resource: Multiple Uses of Culture Under Conditions of Transnational Neoliberalism. PhD. Thesis: York University Ontario, 2013.

ROSA, C. Brazilian Bodies and Their Choreographies of Identification. U.K.: Palgrave Macmillan, 2015.

SHELLER, M. y URRY, J. "The New Mobilities Paradigm". Environment and Planning A. 38 (2): 207-26, 2006.

SALAZAR, N. "Introduction: Keywords of Mobility". In: SALAZAR, N. Y JAYRAM, K. Keywords of Mobility: Critical Engagements. New York: Berghahn Books, 1-12, 2016.

TURNER, V. "El centro está afuera: La jornada del peregrino". Revista Maguaré 23: 15-64, 2009

STOLLER, Paul, y Olkes, C. Sorcery's Shadow: A Memoir of Apprenticeship among the Songhay of Niger. Chicago: University of Chicago Press, 1987.

URRY, J. Mobilities. Cambridge: Polity, 2007.

VIVEIROS DE CASTRO, E. The Relative Native: Essays on Indigenous Conceptual Worlds. WAGNER, R. Chicago: Hau Books. 2015.

WAGNER, R. The Invention of Culture. Revised and expanded edition. Chicago: University of Chicago Press, 1981.

WESOLOWSKI, K. "Professionalizing Capoeira: The Politics of Play in Twenty-first Century Brazil". Latin American Perspective 183 Vol.39 Número 2: 82-92, 2012. 